\title{
Complete disease remission in a TP53 and KRAS co-mutated brain oligometastatic lung cancer patient after immuno-chemotherapy and surgical resection: a case report
}

\author{
Shuai Zhu ${ }^{1,2 \#}$, Shikang Zhao ${ }^{1,2 \#}$, Qian Zhang ${ }^{3 \#}$, Shuo Li $^{3}$, Dian Ren ${ }^{1,2}$, Fan Ren ${ }^{1,2}$, Lingling Zu ${ }^{2}$, \\ Yanye Wang ${ }^{1,2}$, Xi Lei ${ }^{1,2}$, Ning Zhou ${ }^{1,2}$, Tao Shi ${ }^{4}$, Dongbo Xu ${ }^{5}$, Gang Chen ${ }^{1}$, Wan-Teck Lim ${ }^{6}$, \\ Raffaele Giusti ${ }^{7}$, Abraham J. Wu ${ }^{8}$, Song $\mathrm{Xu}^{1,2 \wedge}$ \\ ${ }^{1}$ Department of Lung Cancer Surgery, Tianjin Medical University General Hospital, Tianjin, China; ${ }^{2}$ Tianjin Key Laboratory of Lung Cancer \\ Metastasis and Tumor Microenvironment, Lung Cancer Institute, Tianjin Medical University General Hospital, Tianjin, China; ${ }^{3}$ Department of \\ Respiratory and Critical Care, Tianjin Medical University General Hospital, Tianjin, China; ${ }^{4}$ Precision Medicine Center, Tianjin Medical University \\ General Hospital, Tianjin, China; ${ }^{5}$ Department of Pathology, Tianjin Medical University Cancer Institute and Hospital, Tianjin, China; ${ }^{6}$ Division \\ of Medical Oncology, National Cancer Centre Singapore, Singapore; ${ }^{7}$ Medical Oncology Unit, Sant'Andrea Hospital of Rome, Rome, Italy; \\ ${ }^{8}$ Department of Radiation Oncology, Memorial Sloan Kettering Cancer Center, New York, NY, USA \\ \#These authors contributed equally to this work. \\ Correspondence to: Song Xu, MD, PhD. Department of Lung Cancer Surgery, Lung Cancer Institute, Tianjin Medical University General Hospital, \\ No.154 Anshan Road, Heping Distritct, Tianjin 300052, China. Email: xusong198@hotmail.com.
}

\begin{abstract}
Lung cancer is the most common primary malignancy and tends to metastasize to the brain. A multimodal approach, including systematic therapy (targeted therapy, chemotherapy, immunotherapy) and local consolidative therapy (surgical intervention, radiation therapy, ablation therapy), is essential for treatment of oligometastatic lung cancer. The systemic immunotherapy has been shown to increase response rate and survival, which then has the potential benefit of making localized treatment more feasible for some cases of oligometastatic cancer. We present a 62-year-old male with stage IVB lung adenocarcinoma with five metastases in the brain. Molecular testing exhibited KRAS and TP53 co-mutation, with negative PDL1 expression. The patient received six cycles of platinum-based chemotherapy plus pembrolizumab and minimally invasive lobectomy, followed by maintenance therapy with pemetrexed and pembrolizumab. The patient achieved complete disease remission, with no sign of recurrence for 22 months post-treatment. Moreover, we investigated PD-L1 expression and infiltration of immunological cells in biopsy tissue and surgical specimen prior to and after immuno-chemotherapy using multiple immunohistochemistry stains. The different infiltration levels of immune cells for TP53 and KRAS co-mutation were also explored using The Cancer Genome Atlas (TCGA) database and Cell type Identification By Estimating Relative Subsets Of RNA Transcripts (CIBERSORT). To our knowledge, this is the first reported case in which a brain oligometastatic non-small cell lung carcinoma (NSCLC) patient has achieved a complete response after immuno-chemotherapy plus local surgical resection.
\end{abstract}

Keywords: Oligometastatic; non-small cell lung carcinoma (NSCLC); immuno-chemotherapy; surgical resection; case report

Submitted Dec 22, 2020. Accepted for publication May 21, 2021.

doi: $10.21037 /$ tlcr-21-380

View this article at: http://dx.doi.org/10.21037/tlcr-21-380

$\wedge$ ORCID: 0000-0001-6153-387X. 


\section{Introduction}

Advanced non-small cell lung carcinoma (NSCLC) has a poor prognosis, with a 5 -year overall survival (OS) rate of approximately $5 \%$. Targeted therapy could contribute to improved survival for patients with actionable driver gene mutations but has no impact in driver gene-negative patients. With the application of immune checkpoint inhibitors (ICIs), the 5-year OS rate of advanced EGFR and ALK wild-type NSCLC has considerably improved. Results of a randomized phase I trial (KEYNOTE-001) suggest that pembrolizumab monotherapy provided durable antitumor activity, with a $23.2 \%$ and $15.5 \%$ increase in 5 -year OS rates for treatment-naïve and previously treated advanced NSCLC patients, respectively. Furthermore, a randomized phase III trial (KEYNOTE-024) also demonstrated that the 5-year OS rate increased to $31.9 \%$ with pembrolizumab monotherapy in patients with advanced NSCLC with PDL1 expression greater than $50 \%$.

Oligometastasis disease, which is defined as the intermediate stage between locally advanced and widely disseminated disease, was proposed by Hellman and Weichselbaum in 1995 (1). The concept of oligometastatic lung cancer has been controversial; however, in 2019 a consensus statement was released with reference to the possibility of offering radical-intent treatment strategies. This consensus definition for oligometastatic lung cancer allowed a maximum of five metastases in up to three organs, without the presence of diffuse serosal metastases or bone marrow involvement (2). Furthermore, the incidence rate was about $4.0 \%$ for brain oligometastasis in NSCLC patients. Multimodal treatment is essential for oligometastatic lung cancer patients. A multicenter, phase II randomized study demonstrated that local consolidative therapy, in addition to systemic therapy, could significantly prolong the progression-free survival (PFS) and OS compared to systemic therapy alone (3). As a radical treatment, surgical resection is the most established local consolidative therapy, with previous studies showing that surgical therapy in oligometastatic lung cancer contributed to significantly better 5-year OS in various multimodal approaches (4). Combinations of ICI and chemotherapy may markedly increase response rate and survival which might allow oligometastatic lung cancer patients to become eligible for localized treatment modalities. Mutations of TP53 and KRAS are common in NSCLC patients, and previous study conducted the presence of TP53 and KRAS co-mutation has also been associated with response to checkpoint inhibitors. This report describes a brain oligometastatic lung cancer patient with TP53 and $K R A S$ co-mutation who achieved a complete response after systemic immuno-chemotherapy and local surgical resection. As we known, this is the first reported case in which a brain oligometastatic NSCLC patient has achieved a complete response after immuno-chemotherapy plus local surgical resection. We present the following article in accordance with the CARE reporting checklist (available at http://dx.doi.org/10.21037/tlcr-21-380).

\section{Case presentation}

A 62-year-old male with a 40-year smoking history was hospitalized following a one-month history of bloody sputum. Enhanced computed tomography (CT) indicated a mass with pleural invasion in the right upper lobe $3.5 \mathrm{~cm}$ $\times 3.3 \mathrm{~cm}$ in size (Figure 1A). Enhanced magnetic resonance imaging (MRI) showed five metastases $(6.2 \mathrm{~mm}$ in right temporal lobe, $6.4 \mathrm{~mm}$ in right frontal lobe, $5.7 \mathrm{~mm}$ in left hippocampus, $9.5 \mathrm{~mm}$ in left parietal lobe and $17.9 \mathrm{~mm}$ in left temporal lobe) in the brain without edema as shown in Figure $1 B$. He has no any neurological symptoms. A CT-guided percutaneous biopsy for the lung mass was performed. Pathological examination showed that the right lung lesion was an invasive lung adenocarcinoma. The patient was diagnosed with oligometastatic Stage IVB lung adenocarcinoma (T2aN0M1c, IVB, AJCC 8th). We further performed next-generation sequencing with 68 cancerrelated genes (Burning Rock Biotech, Guangzhou, China) where KRAS (p.Gly13Asp, 8.12\%) and TP53 (p.Thr125Pro, $7.35 \%)$ mutations were detected. Immunohistochemistry with PD-L1 IHC 22C3 pharmDx assay (Dako) for PD-L1 expression was negative.

After the patient's consent, the patient received four cycles of pemetrexed $\left(500 \mathrm{mg} / \mathrm{m}^{2}, \mathrm{~d} 1\right)$, nedaplatin $\left(75 \mathrm{mg} / \mathrm{m}^{2}, \mathrm{~d} 1\right)$ and pembrolizumab (100 mg, d1) every 21 days. After four cycles of systemic immuno-chemotherapy, a brain MRI indicated that three metastatic tumors had completely disappeared and the remaining two metastases had a marked partial response (PR) (Figure 1B). After four cycles of systemic immunochemotherapy, brain MRI indicated that three metastatic tumors had completely disappeared and the remaining two metastases had a marked PR status (Figure 1B). Chest CT showed that the right upper lobe lesion had approximately shrunk down by $25.7 \%$ in size but had not yet reached PR by Response Evaluation Criteria in Solid Tumors (RECIST) (Figure 1A). The only side effects during 
A

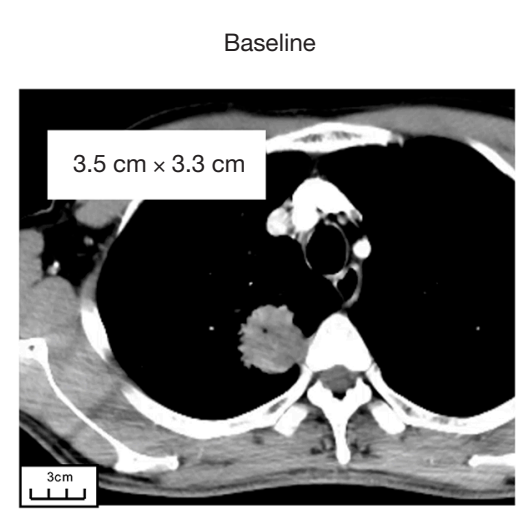

After two cycles

immuno-chemotherapy

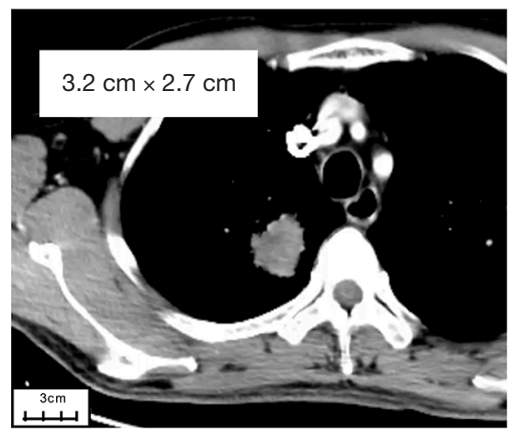

SD
After four cycles

immuno-chemotherapy

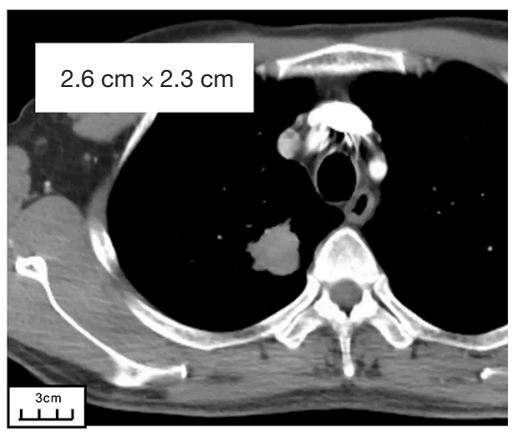

PR not reached

$(-25.7 \%)$

B

Baseline

After 2 cycles

immuno-chemotherapy

After 4 cycles immuno-chemotherapy

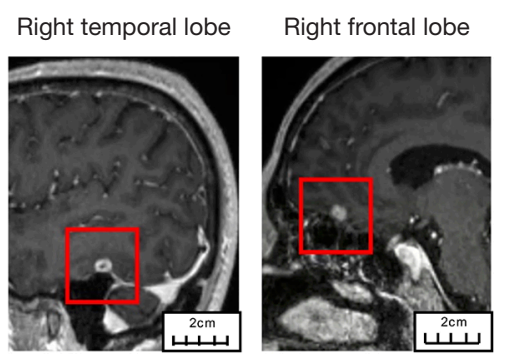

Left hippocampus

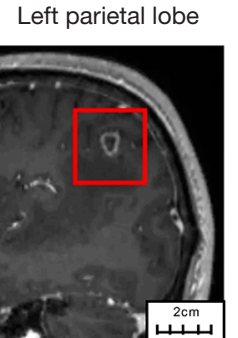

Left temporal lobe
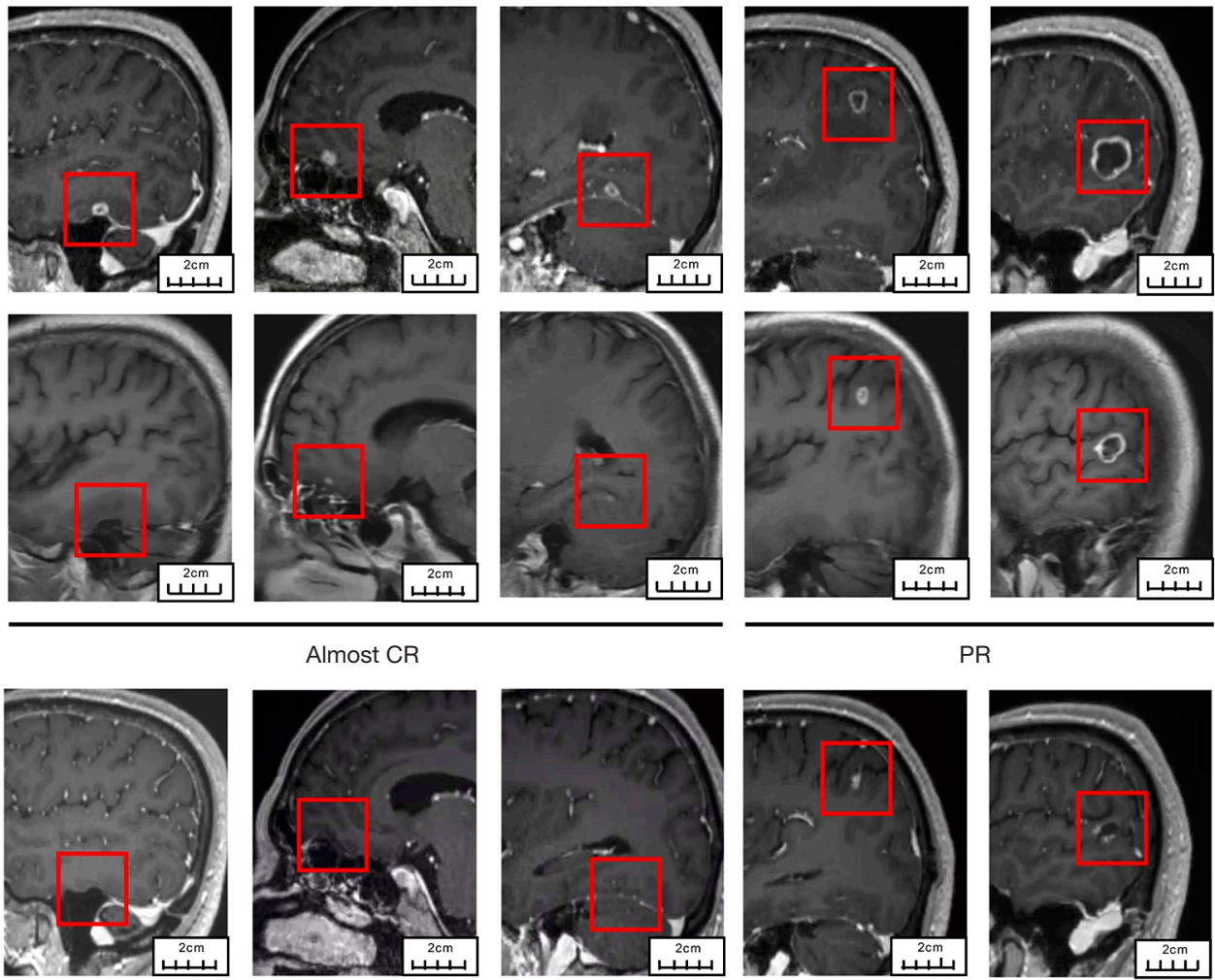

$2 \mathrm{~cm}$

CR

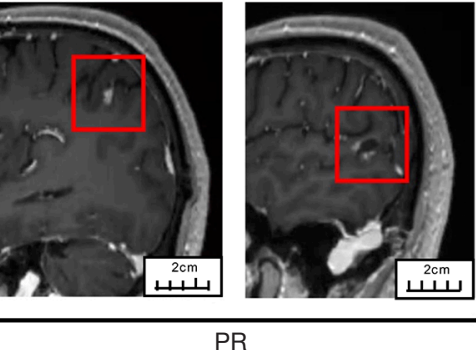




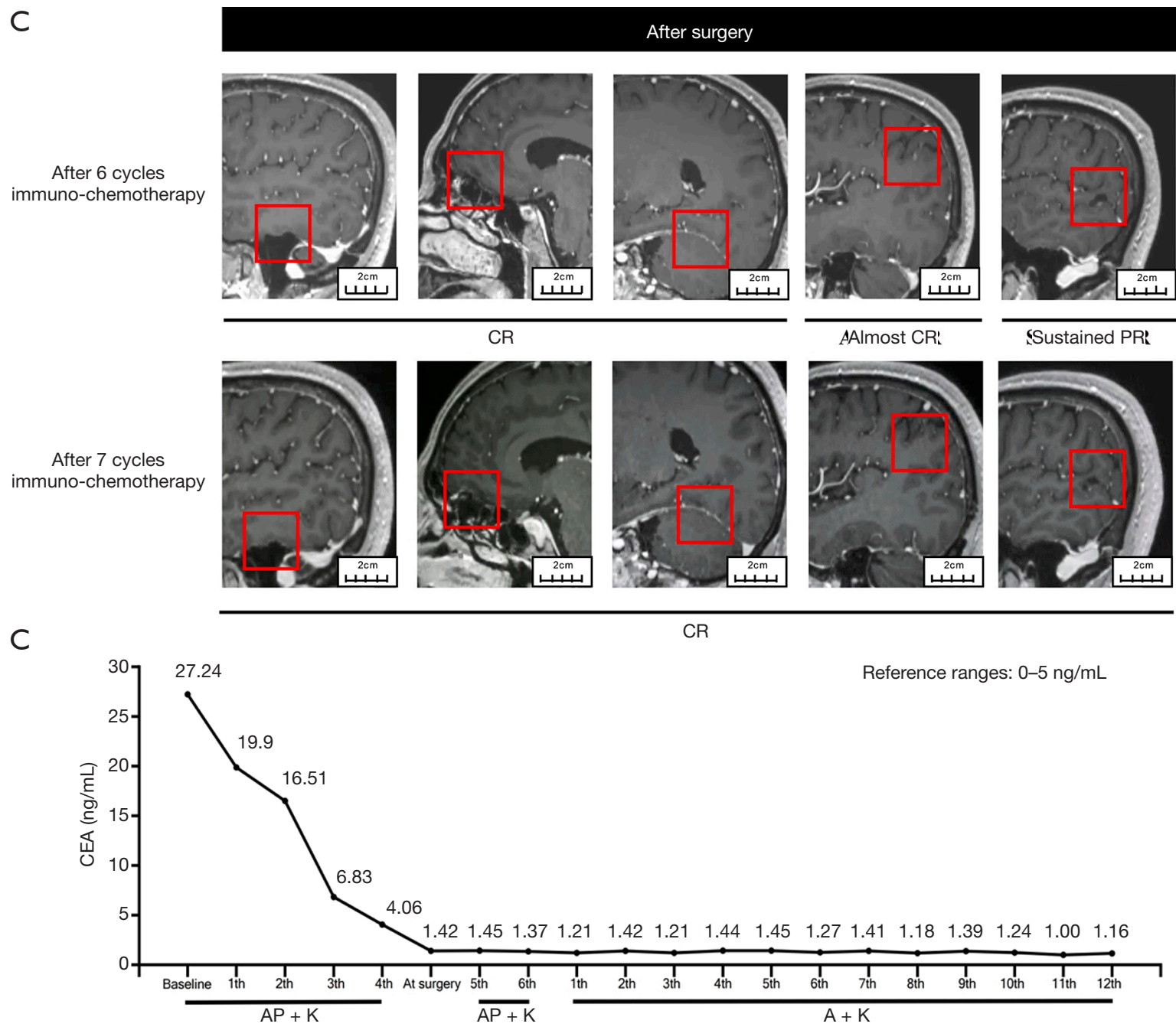

Figure 1 The change of radiological images and serum CEA. (A) Enhanced CT of chest; (B) enhanced MRI of brain; (C) dynamic alteration of serum CEA. Red rectangle indicates the brain metastases of patients. CEA, carcinoembryonic antigen; SD, stable disease; PR, partial response; A, Alimta (Pemetrexed); AP, Alimta (Pemetrexed) + Nedaplatin; K, Keytruda (Pembrolizumab); MRI, magnetic resonance imaging.

treatment were fatigue and gastrointestinal discomfort. The serum carcinoembryonic antigen (CEA) levels had significantly decreased to the normal range (Figure 1C). After careful multidisciplinary discussion and obtaining patient consent, we performed video-assisted right upper lung lobectomy plus regional lymphadenectomy. Pathologic results revealed that the pathological remission rate was $60 \%$ and all lymph node stations were negative. As shown in Figure $2 A$, widespread fibrosis and lymphocyte infiltration could be seen in the surgical specimen after immunochemotherapy. The patient received another two cycles of platinum-based immuno-chemotherapy after surgery, followed by maintenance therapy with pemetrexed and pembrolizumab. After seven cycles of maintenance therapy, MRI showed that all metastases in the brain could not be detected (Figure 1B); thus, the patient achieved a complete disease remission after systemic immuno-chemotherapy plus local surgical resection, with no sign of recurrence after 22 months. This study was approved by Ethics Committee of Tianjin Medical University General Hospital. All procedures performed in studies involving human participants were in accordance with the ethical standards of the institutional and/or national research committee(s) and with the Helsinki Declaration (as revised in 2013). 
A

Pre-treatment biopsy

Surgical specimen
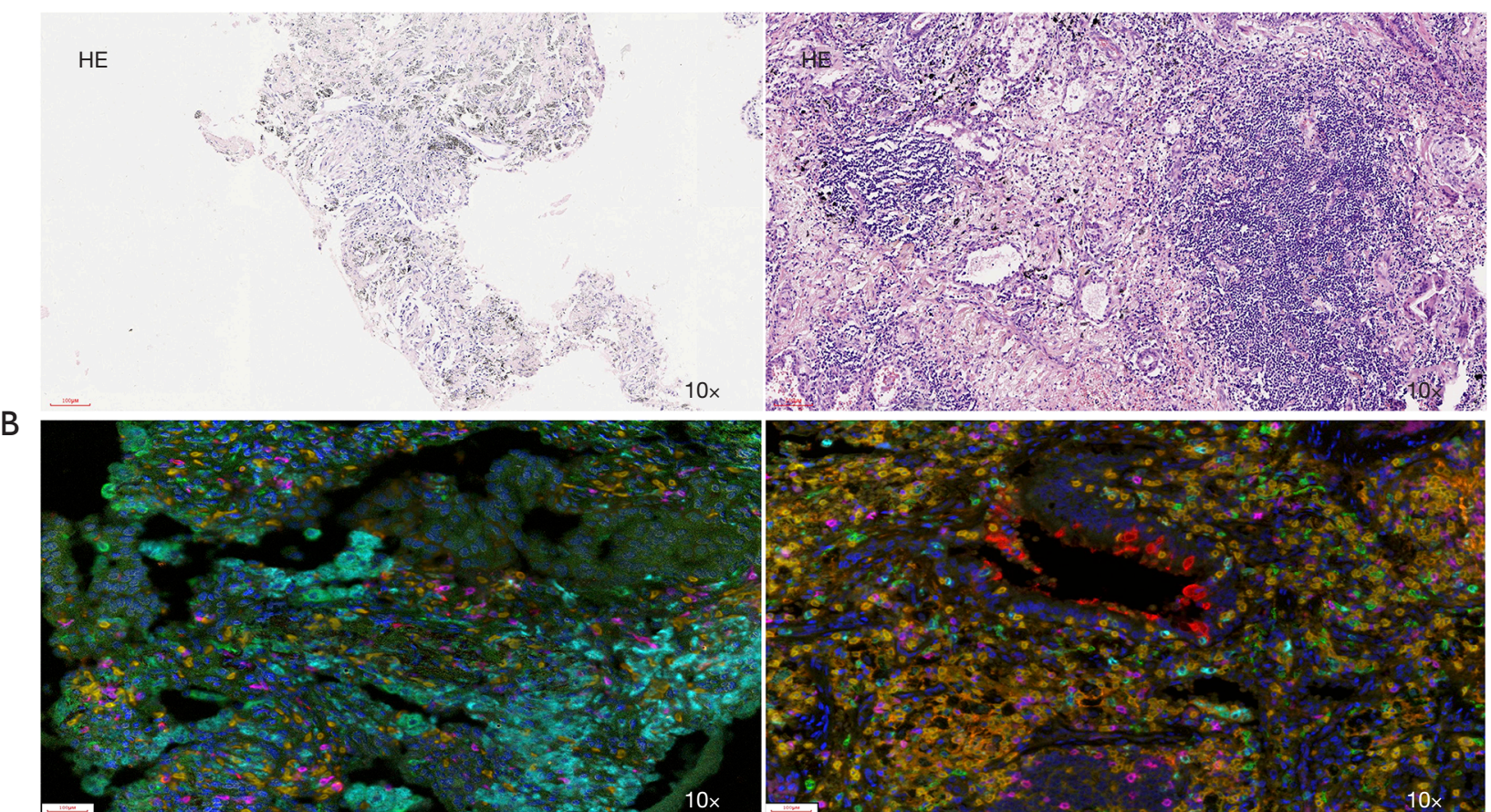

CD163/CD8/PDL1/CD57/CD68/PD1

C
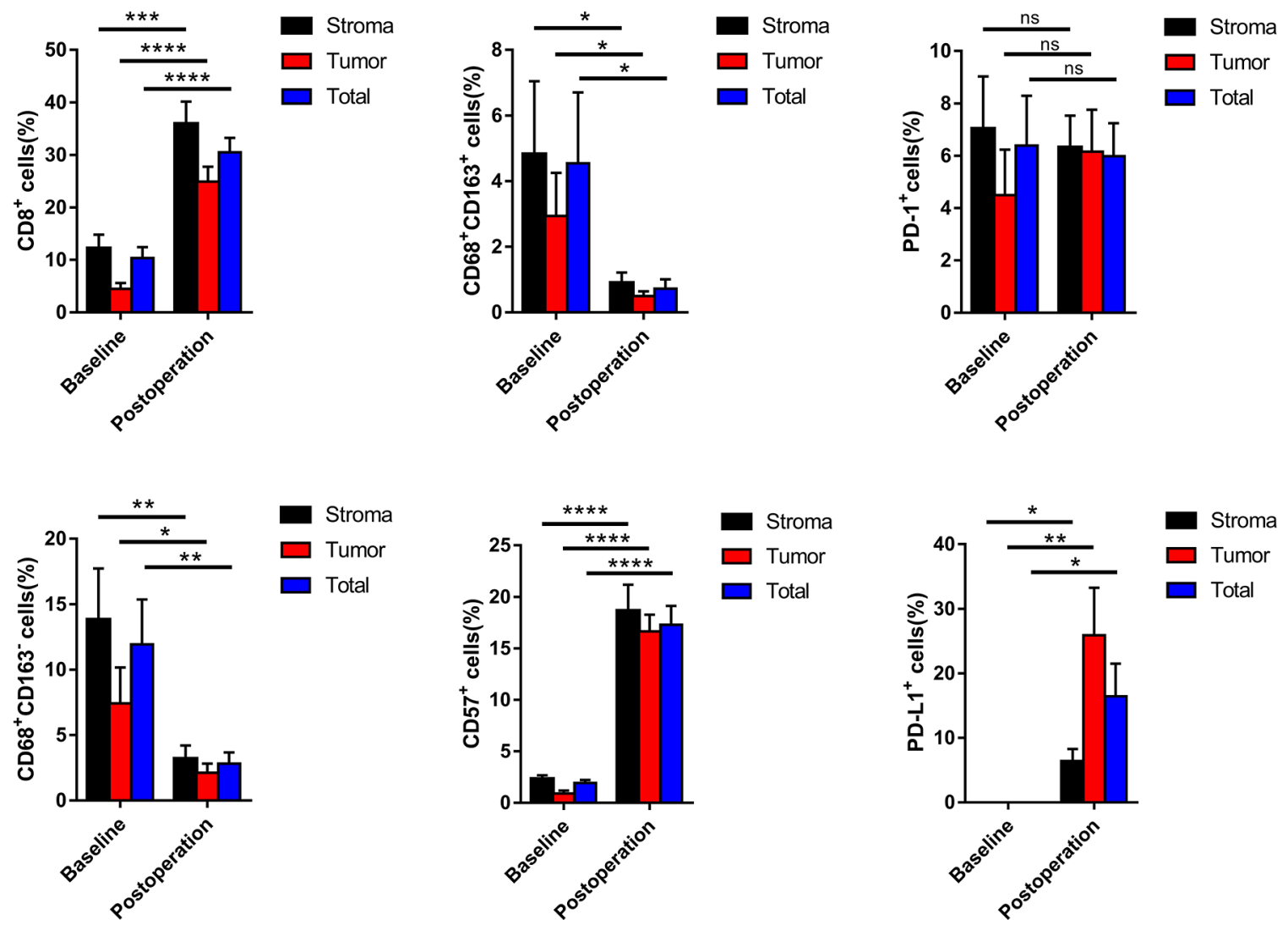


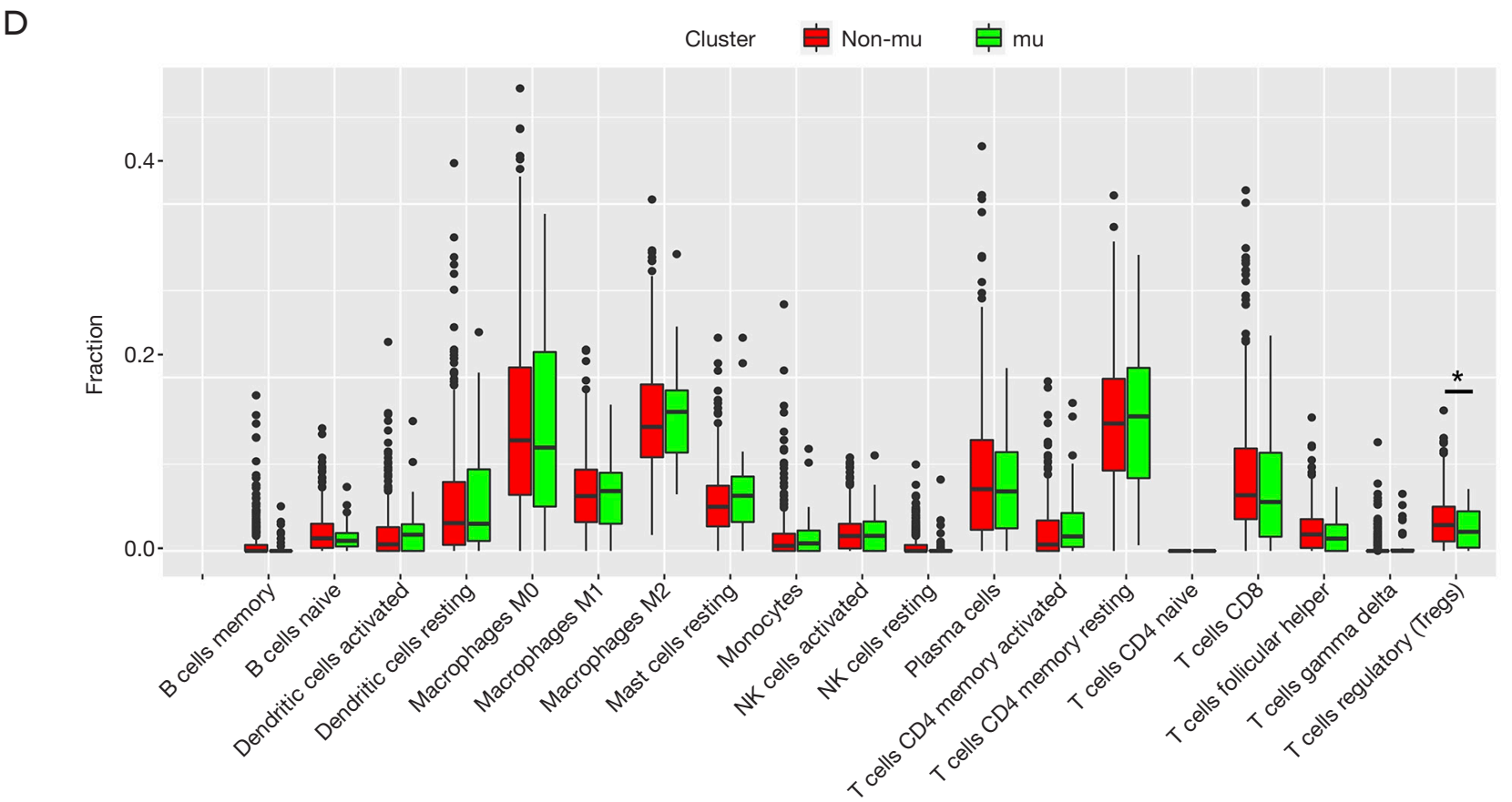

Figure 2 Comprehensive pathological and immunological evaluation. (A) HE staining. Fibrosis and lymphocyte infiltration were seen on the surgical specimen after immuno-chemotherapy; (B) multiple immunohistochemistry staining on CD163, CD8, PD-L1, CD57, CD68 and PD-1 before and after immuno-chemotherapy. The multiplex IHC platform, which can detect the expression of multiple markers in a single section, was utilized to measure the expression of PD-1/PD-L1 and immune cell infiltration. The immune markers included CD8 ${ }^{+}$T-cells, CD $68^{+}$macrophages (M1), CD $68^{+} \mathrm{CD} 163^{+}$macrophages $(\mathrm{M} 2)$ and $\mathrm{CD} 57^{+}$natural killer (NK) cells. Slides were scanned using PerkinElmer Vectra (Vectra 3.0.5; PerkinElmer, Massachusetts, USA). The percentage of positively stained cells in all nucleated cells was counted; (C) quantitative analysis for staining data; (D) box plots for the correlation of TP53 and KRAS co-mutation and non-TP53 and KRAS mutation with different immune cell infiltration levels using TCGA database and CIBERSORT. *, $\mathrm{P}<0.05$; **, $\mathrm{P}<0.01$; ***, $\mathrm{P}<0.001$; ***, $\mathrm{P}<0.0001$. ns, not significant; TCGA, The Cancer Genome Atlas; CIBERSORT, Cell type Identification By Estimating Relative Subsets Of RNA Transcripts.

Written informed consent was provided by the patient for specimen collection, genetic testing, use of this information for research purposes, and publication of the case report.

\section{Discussion}

As for advanced NSCLC patients, specifically for oligometastatic NSCLC patients, systematic therapy followed with local consolidative therapy could lead to a better PFS and OS according to the previous studies. To our knowledge, this is the first case in which a brain oligometastatic NSCLC patient has achieved a complete response after immuno-chemotherapy and complete surgical resection of the lung primary. Although long-term follow-up is necessary to evaluate the therapeutic effect of this treatment strategy, the PFS has been approximately two years. Since the best response for immunotherapy is complete response, and treatment was well-tolerated, previous evidence would suggest this patient may have good long-term survival (5). We would like to highlight possible reasons in this patient for the excellent and durable response despite baseline PD-L1 expression of $0 \%$.

Previous data has indicated that ICI activity in cancer patients is linked to the magnitude of T-cell response within the tumor microenvironment (6). Schaer et al. have also demonstrated that pemetrexed chemotherapy could promote T-cell trafficking and infiltration of the tumor, with consequent enhanced effects of cancer immunotherapy (7). However, there are few studies concerning alteration of the immune microenvironment after systemic immunochemotherapy. As illustrated in Figure $2 B, C$, PDL1 expression was dramatically increased, along with 
macrophage regression in the tumor after immunochemotherapy. It was also found that infiltration of $\mathrm{CD} 8^{+}$ and NK cells was upregulated after immuno-chemotherapy. These results suggest that the underlying mechanism for disease remission in this patient was through immune activation enhanced by pemetrexed based chemotherapy. The dynamic nature of the PD-L1 upregulation with chemotherapy shown in this patient also illustrates the poor utility of static PD-L1 expression at baseline as a sole biomarker for use of ICI, and argues for better biomarkers for selection for treatment.

The presence of TP53 and KRAS co-mutation has also been associated with response to checkpoint inhibitors. Fang et al. reported that co-mutations of TP53 and KRAS served as potential biomarkers for response to PD-1 blockade immunotherapy in squamous-cell NSCLC (8). Dong et al. observed that TP53 or KRAS mutation in lung adenocarcinoma, especially those with co-occurring TP53/ KRAS mutations, showed increased expression of PD-L1 and $\mathrm{CD} 8^{+} \mathrm{T}$ cells, along with marked clinical sensitivity to PD-1 inhibitors (9). In this study, we also investigated immune cell infiltration in TP53 and KRAS co-mutation in NSCLC using TCGA database and Cell type Identification By Estimating Relative Subsets Of RNA Transcripts (CIBERSORT). As shown in Figure 2D, TP53 and KRAS co-mutation was negatively correlated with infiltration of $\mathrm{T}$ regulatory cells (Tregs), which have been established as playing a role in the suppression of antitumor immunity (10). These findings indicate that TP53 and KRAS co-mutation constitute a "hot" immune microenvironment favoring antiPD-1/PD-L1 immunotherapy.

In conclusion, we present a brain oligometastatic NSCLC patient with complete response after systemic immuno-chemotherapy and local surgical resection. This is a new mode of treatment for certain NSCLC patients with oligometastatic disease, which may achieve a better curative effect and survival. However, due to the complexity of the clinical diagnosis and individual difference, it is still uncertain whether this multimodality treatment regimen is suitable for all the oligometastatic NSCLC patients. Therefore, further investigation is worthwhile in this population, especially for patients with certain genetic mutations.

\section{Acknowledgments}

The authors appreciate the academic support from AME Lung Cancer Collaborative Group.
Funding: The present study was funded by the National Natural Science Foundation of China (No. 81772464).

\section{Footnote}

Reporting Checklist: The authors have completed the CARE reporting checklist. Available at http://dx.doi.org/10.21037/ tlcr-21-380

Conflicts of Interest: All authors have completed the ICMJE uniform disclosure form (available at http://dx.doi. org/10.21037/tlcr-21-380). The authors have no conflicts of interest to declare.

Ethical Statement: The authors are accountable for all aspects of the work in ensuring that questions related to the accuracy or integrity of any part of the work are appropriately investigated and resolved. This study was approved by Ethics Committee of Tianjin Medical University General Hospital. All procedures performed in studies involving human participants were in accordance with the ethical standards of the institutional and/or national research committee(s) and with the Helsinki Declaration (as revised in 2013). Written informed consent was provided by the patient for specimen collection, genetic testing, use of this information for research purposes, and publication of the case report.

Open Access Statement: This is an Open Access article distributed in accordance with the Creative Commons Attribution-NonCommercial-NoDerivs 4.0 International License (CC BY-NC-ND 4.0), which permits the noncommercial replication and distribution of the article with the strict proviso that no changes or edits are made and the original work is properly cited (including links to both the formal publication through the relevant DOI and the license). See: https://creativecommons.org/licenses/by-nc-nd/4.0/.

\section{References}

1. Hellman S, Weichselbaum RR. Oligometastases. J Clin Oncol 1995;13:8-10.

2. Dingemans AC, Hendriks LEL, Berghmans T, et al. Definition of Synchronous Oligometastatic Non-Small Cell Lung Cancer-A Consensus Report. J Thorac Oncol 2019;14:2109-19.

3. Gomez DR, Tang C, Zhang J, et al. Local Consolidative Therapy Vs. Maintenance Therapy or Observation for 
Patients With Oligometastatic Non-Small-Cell Lung Cancer: Long-Term Results of a Multi-Institutional, Phase II, Randomized Study. J Clin Oncol 2019;37:1558-65.

4. David EA, Clark JM, Cooke DT, et al. The Role of Thoracic Surgery in the Therapeutic Management of Metastatic Non-Small Cell Lung Cancer. J Thorac Oncol 2017;12:1636-45.

5. Gauci ML, Lanoy E, Champiat S, et al. Long-Term Survival in Patients Responding to Anti-PD-1/PDL1 Therapy and Disease Outcome upon Treatment Discontinuation. Clin Cancer Res 2019;25:946-56.

6. Paijens ST, Vledder A, de Bruyn M, Nijman HW. Tumorinfiltrating lymphocytes in the immunotherapy era. Cell Mol Immunol 2021;18:842-59.

7. Schaer DA, Geeganage S, Amaladas N, et al. The Folate

Cite this article as: Zhu S, Zhao S, Zhang Q, Li S, Ren D, Ren F, Zu L, Wang Y, Lei X, Zhou N, Shi T, Xu D, Chen G, Lim WT, Giusti R, Wu AJ, Xu S. Complete disease remission in a TP53 and KRAS co-mutated brain oligometastatic lung cancer patient after immuno-chemotherapy and surgical resection: a case report. Transl Lung Cancer Res 2021;10(5):2298-2305. doi: $10.21037 /$ tlcr-21-380
Pathway Inhibitor Pemetrexed Pleiotropically Enhances Effects of Cancer Immunotherapy. Clin Cancer Res 2019;25:7175-88.

8. Fang C, Zhang C, Zhao WQ, et al. Co-mutations of TP53 and KRAS serve as potential biomarkers for immune checkpoint blockade in squamous-cell non-small cell lung cancer: a case report. BMC Med Genomics 2019;12:136.

9. Dong ZY, Zhong WZ, Zhang XC, et al. Potential Predictive Value of TP53 and KRAS Mutation Status for Response to PD-1 Blockade Immunotherapy in Lung Adenocarcinoma. Clin Cancer Res 2017;23:3012-24.

10. Persa E, Balogh A, Sáfrány G, et al. The effect of ionizing radiation on regulatory $T$ cells in health and disease. Cancer Lett 2015;368:252-61. 